\title{
A GENERALIZED APPROXIMATION THEOREM FOR DEDEKIND DOMAINS
}

\section{LUTHER CLABORN}

It is well known that a Dedekind domain $A$ with a finite number of prime ideals is a principal ideal domain. A reasonable generalization of this result would be: If $A$ is a Dedekind domain and $S$ is the set of prime ideals of $A$, then card $S<$ card $A$ implies that $A$ is a principal ideal domain.

In fact, this latter statement is false; see [1]. But it is true that if (card $S)^{\aleph_{0}}<$ card $A$, then $A$ is a principal ideal domain. A proof is given in the present article of a slight generalization (analogous to the weak approximation theorem) of this result.

Before proceeding to this result, we give a proposition that displays a large class of examples for which the stronger assertion of the first paragraph is valid. We will use the phrase "Let $A, S$ be a Dedekind domain" rather than "Let $A$ be a Dedekind domain, and let $S$ be the set of prime ideals of $A$ " for the balance of the article. Also, if $P$ is a prime ideal of $A$, then $v_{p}$ will denote the normed valuation going with the prime ideal $P$.

Proposition. Let $A, S$ be a Dedekind domain and suppose that $A$ contains a field $F$ such that $\operatorname{card} F=\operatorname{card} A$. Suppose that $\operatorname{card} S<\operatorname{card} A$. Then $A$ is a principal ideal domain.

Proof. Let $P$ be in $S$; choose $\pi$ and $\sigma$ in $A$ such that $v_{p}(\pi)=1$, $v_{p}(\sigma)=2$ and $P=(\pi, \sigma)$. Consider the set of elements $\pi+f \sigma$ for $f$ in $F$. For all $f$, we have $v_{p}(\pi+f \sigma)=1$. If $P$ is not principal, then for each $f$, there must be a $Q \neq P$ in $S$ such that $\pi+f \sigma$ is in $Q$. Since card $F$ $>$ card $S$, there will be an $f$ and $f^{\prime}$ in $F$ such that $\pi+f \sigma$ and $\pi+f^{\prime} \sigma$ are in the same prime ideal $Q \neq P$ of $S$. But then $\left(f-f^{\prime}\right) \sigma$ is in $Q$, so $\sigma$ is $Q$, forcing also $\pi$ in $Q$. This implies that $P=(\pi, \sigma)$ is contained in $Q$, which is a contradiction.

REMARK. If $T$ is a subset of $S, P$ is a prime ideal in $T$, and there is an element $a_{P}$ of $A$ such that $v_{Q}\left(a_{P}\right)=\delta_{P, Q}$ for all $Q$ in $T$, then we will say that $P$ is principal with respect to $T$.

Theorem. Let $A, S$ be a Dedekind domain and let $T$ be a subset of $S$ such that not every prime ideal of $T$ is principal with respect to $T$. Then card $A \leqq(\operatorname{card} T)^{\aleph_{0}}$.

Received by the editors May 16, 1966. 
Proof. If $T$ is a finite set, the weak approximation theorem handles the situation. If any prime ideal $P$ of $S$ is such that $A / P$ is finite, then imbedding $A$ in its $P$-adic completion shows that card $A$ $\leqq(\operatorname{card} A / P)^{\aleph_{0}} \leqq(\operatorname{card} T)^{\aleph_{0}}$; so we may assume that $A / P$ is infinite for any $P$ in $S$.

We first show that there is a subset $S^{\prime}$ of $S$ such that $T$ is contained in $S^{\prime}$, card $T=$ card $S^{\prime}$, and $S^{\prime}$ contains an infinite number of prime ideals which are not principal with respect to $S^{\prime}$. If $T$ will not work, let $P_{1}, \cdots, P_{k}$ be the prime ideals of $T$ which are not principal with respect to $T$. For each $Q$ in $T-\left\{P_{1}, \cdots, P_{k}\right\}$ let $a_{Q}$ be a generator of $Q$ with respect to $T$. Let $W$ be the set of prime ideals of $S-T$ which contain an $a_{Q}$ for some $Q$ in $T-\left\{P_{1}, \cdots, P_{k}\right\}$. Set $T^{\prime}=T \cup W$. $P_{1}, \cdots, P_{k}$ are certainly not principal with respect to $T^{\prime}$; further, not all prime ideals $R$ in $W$ can be principal with respect to $T^{\prime}$. For suppose they are, and let $a_{R}(R$ in $W)$ be a generator for $R$ with respect to $T^{\prime}$. Choose $x$ in $A$ such that $v_{p_{1}}(x)=1, v_{p_{i}}(x)=0$ for $i=2, \cdots, k$. Multiplying $x$ by appropriate negative powers of the $a_{Q}\left(Q\right.$ in $\left.T-\left\{P_{1}, \cdots, P_{k}\right\}\right)$ yields an element $y$ in the quotient field of $A$ such that $v_{p_{1}}(y)=1, v_{p_{1}}(y)=0$ for $i=2, \cdots, k$, and $v_{Q}(y)=0$ for $Q$ in $T-\left\{P_{1}, \cdots, P_{k}\right\}$. But $y$ will have negative values only for certain of the $v_{R}(R$ in $W)$. Multiplying by appropriate positive powers of the $a_{R}(R$ in $W)$ produces an element which generates $P_{1}$ with respect to $T^{\prime}$ (hence with respect to $T$ ) and gives a contradiction.

Inductively, set $T_{1}=T^{\prime}$ and $T_{n}=\left(T_{n-1}\right)^{\prime}$. Then $U_{1}^{\infty} T_{n}$ works.

We will now assume that $T$ contains an infinite sequence of primes $P_{1}, P_{2}, \cdots$, which are not principal with respect to $T$. We will let $v_{i}$ denote the valuation going with $P_{i}$. Choose $\pi_{1}$ and $\sigma_{1}$ such that $v_{1}\left(\pi_{1}\right)=1, v_{1}\left(\sigma_{1}\right)=2$ and $P_{1}=\left(\pi_{1}, \sigma_{1}\right)$. Delete from the list the $P_{i}(i>1)$ which contain $\sigma_{1}$ and renumber the remaining primes in their original order. Choose $\pi_{2}$ and $\sigma_{2}$ such that $v_{2}\left(\pi_{2}\right)=1, v_{2}\left(\sigma_{2}\right)=2, P_{2}=\left(\pi_{2}, \sigma_{2}\right)$ and $\pi_{2} / \sigma_{2} \neq \pi_{1} / \sigma_{1}$. Delete from the list the $P_{i}(i>2)$ which contain $\sigma_{1}$ or $\sigma_{2}$ or for which $v_{i}\left(\pi_{2} / \sigma_{2}-\pi_{1} / \sigma_{1}\right)>0$. Inductively, choose $\pi_{j}$ and $\sigma_{j}$ such that $v_{j}\left(\pi_{j}\right)=1, v_{j}\left(\sigma_{j}\right)=2, P_{j}=\left(\pi_{j}, \sigma_{j}\right)$ and also subject to the condition: if $k<j$, then $\pi_{j} / \sigma_{j} \not \equiv \pi_{k} / \sigma_{k}$ modulo any of the prime ideals $Q$ of $S$ for which $v_{Q}\left(\pi_{m} / \sigma_{m}-\pi_{n} / \sigma_{n}\right)$ is positive with $m, n<j$ (this of course provided $\left.v_{Q}\left(\pi_{k} / \sigma_{k}\right) \geqq 0\right)$. This is possible since $A / P$ is infinite for all $P$ in $S$ and since $v_{j}$ is not positive at $\pi_{m} / \sigma_{m}-\pi_{n} / \sigma_{n}$ with $m, n<j$. Then delete the $P_{i}(i>j)$ which contain any of $\sigma_{1}, \cdots, \sigma_{j}$ or for which $v_{i}\left(\pi_{m} / \sigma_{m}-\pi_{n} / \sigma_{n}\right)>0$ with $m, n \leqq j$ and renumber.

Let $a$ be an element of $A$, and consider the set $\left\{\pi_{i}+a \sigma_{i}\right\}$. For each $i, v_{i}\left(\pi_{i}+a \sigma_{i}\right)=1$, and $P_{i}$ is not principal with respect to $T$, so there is a prime ideal $Q_{i}\left(\not \neq P_{i}\right)$ in $T$ such that $\pi_{i}+a \sigma_{i}$ is in $Q_{i}$. Making 
some choice for each $i$, let $f_{a}: i \rightarrow Q_{i}$ be the map induced by $a$. The image of $f_{a}$ is infinite. If not, then there is a finite subset $R_{1}, \cdots, R_{m}$ of $T$ such that $\pi_{i}+a \sigma_{i}$ is contained in one of these for each $i$. Suppose that for an infinite number of $i, \pi_{i}+a \sigma_{i}$ is in $R_{1}$. If $R_{1}$ is a $P_{n}$ for some $n$, choose $p, q, r$ such that $p>q>r>n$ with $\pi_{p}+a \sigma_{p}, \pi_{q}+a \sigma_{q}, \pi_{r}+a \sigma_{r}$ all in $R_{1}$ (if $R_{1}$ is not in the set $\left\{P_{i}\right\}$, simply choose $p>q>r$ ). If $\sigma_{j}$ is in $R_{1}$ for $j=p, q$, or $r$, then $\pi_{j}$ is also in $R_{1}$ forcing $P_{j}$ to be $R_{1}$ and giving a contradiction. We then have $\pi_{j} / \sigma_{j} \equiv-a\left(R_{1}\right)$ for $j=p, q, r$ and so we get $\pi_{p} / \sigma_{p} \equiv \pi_{q} / \sigma_{q}$ modulo $R_{1}$, but $v_{R_{1}}$ has positive value at $\pi_{q} / \sigma_{q}-\pi_{r} / \sigma_{r}$ which contradicts the construction for the $\pi_{i}$ and $\sigma_{i}$.

If now $f_{a}=f_{b}$, we get that $\pi_{i}+a \sigma_{i}$ and $\pi_{i}+b \sigma_{i}$ are in the same ideal $Q_{i}$ for all $i$. This yields $(a-b) \sigma_{i}$ in $Q_{i}$ for all $i$. We cannot have $\sigma_{i}$ in $Q_{i}$ (else $P_{i}=Q_{i}$ ) so $a-b$ is in $Q_{i}$ for all $i$. Since the set $\left\{Q_{i}\right\}$ is infinite, $a=b$.

Letting $N$ denote the natural numbers, we get that each $a$ in $A$ induces a map $f_{a}: N \rightarrow T$. Since $a \rightarrow f_{a}$ is one-to-one, we have card $A$ $\leqq(\operatorname{card} T)^{\aleph_{0}}$.

Corollary 1 (Generalized Approximation Theorem). Let $A$, $S$ be a Dedekind domain, and let $T$ be a subset of $S$ such that (card $T)^{N_{0}}$ $<$ card A. Given a set of nonnegative integers $\left\{n_{p}\right\}$ for $P$ in $T$ which are almost all zero, there is an element $x$ in $A$ such that $v_{p}(x)=n_{p}$ for all $P$ in $T$.

Corollary 2. If $A, S$ is a Dedekind domain and (card $S)^{\aleph_{0}}<\operatorname{card} A$, then $A$ is a principal ideal domain.

\section{REFERENCES}

1. L. Claborn, Specified relations in the ideal group. II, (to appear).

2. E. Weiss, Algebraic number theory, McGraw-Hill, New York, 1963.

Cornell College and

UNIVERSITY OF ILLINOIS, URBANA 